\title{
Application of bacteriorhodopsin films in an adaptive-focusing schlieren system
}

\author{
John D. Downie
}

\begin{abstract}
The photochromic property of bacteriorhodopsin films is exploited in the application of a focusing schlieren optical system for the visualization of optical phase information. By encoding an image on the film with light of one wavelength and reading out with a different wavelength, the readout beam can effectively see the photographic negative of the original image. The potential advantage of this system over previous focusing schlieren systems is that the updatable nature of the bacteriorhodopsin film allows system adaptation. I discuss two image encoding and readout techniques for the bacteriorhodopsin and use film transmission characteristics to choose the more appropriate method. I demonstrate the system principle with experimental results using argon-ion and $\mathrm{He}-\mathrm{Cd}$ lasers as the two light sources of different wavelengths, and I discuss current limitations to implementation with a white-light source.
\end{abstract}

\section{Introduction}

Schlieren optical systems have long been used to examine and visualize optical-phase or refractiveindex distributions. Examples include basic opticalelement-aberration and radius-of-curvature testing and the visualization of airflow-density variations around airfoils undergoing aerodynamic tests in wind tunnels. There are two basic types of schlieren systems, usually designated as conventional and focusing schlieren systems. Both types essentially operate by detecting and imaging light that is deviated or refracted along the optical path from its nominal course in the absence of phase or refractive-index variations. This is accomplished by appropriate spatial filtering that blocks the undeviated light while allowing the transmission of some refracted light.

We examine here the use of bacteriorhodopsin (BR) films in a focusing schlieren system. BR is an organically derived material that demonstrates several desirable properties for use as a real-time optically addressed spatial light modualtor for optical processing applications. ${ }^{1,2}$ These include photochromism, the optical Kerr effect, and photoinduced anisotropy. Some previously studied applications for BR films include Fourier-plane spatial filtering, ${ }^{3}$ optical limiting, ${ }^{4}$ time-averaged holographic interferometry, ${ }^{5}$ real-time holography for optical correlation, ${ }^{6}$ and

The author is with NASA/Ames Research Center, Mail Stop 269-3, Moffet Field, California 94035.

Received 17 January 1995; revised manuscript received 14 April 1995.

0003-6935/95/266021-08\$06.00/0.

1995 Optical Society of America. nonlinear image transformations for optical image processing. ${ }^{7}$ The real-time photochromic behavior of BR is exploited for the application studied here of focusing schleiren systems. Essentially, the BR film is used as a real-time photographic transparency that behaves as a negative image when the appropriate wavelengths of light are employed. This system potentially offers improved flexibility, adaptivity, and ease of alignment with respect to previous focusing schlieren systems. In particular, it may permit easy adjustments in sensitivity to be made without system modifications or downtime and perhaps also allow implementation in unstructured and changing environments such as the outdoors. In this paper I demonstrate the system principle with experimental results using argon-ion and helium-cadmium $(\mathrm{He}-$ Cd) lasers as two light sources of different wavelengths and discuss current limitations to implementation with a white-light source.

The remainder of this paper is divided into three sections. Section 2 describes the general optical setup of a focusing schlieren system and the specific implementation using a BR film. Section 3 presents experiments and results that successfully demonstrate the proof of principle. Section 4 is a summary of the results and a discussion of the current limitations of the technique.

\section{Focusing Schlieren System with Bacteriorhodopsin Film}

\section{A. Basic Focusing-Schlieren-System Characteristics}

A focusing schlieren system was first proposed and implemented by Burton in 1949. ${ }^{\circ}$ A general schematic of this type of system in shown in Fig. 1. A 


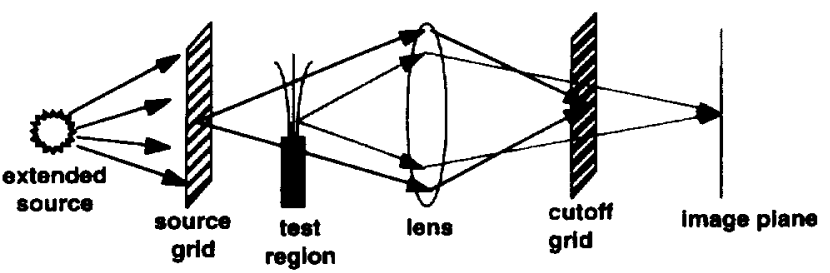

Fig. 1. Schematic of general focusing schlieren optical system with a gas jet being examined in the test region.

light source (generally extended) illuminates a source grid, which is some type of black-and-white periodic pattern such as a grating or a checkerboard. A camera lens images the source grid through the test region containing the phase or refractive-index variations and onto a cutoff grid. The cutoff grid is designed such that it is an exact photographic negative of the source grid (accounting, of course, for the magnification of the camera-lens imaging system). In the absence of any aberrations in the test region, all light originating at the source grid will thus be blocked by the cutoff grid because light areas of the source grid are imaged onto opaque areas of the cutoff grid. In this case the final image plane will be dark. However, if in the test region there are refractiveindex variations that are due to density variations in a fluid flow, for example, then light passing through the affected part of the test region will be refracted from its normal course. Some of that light will not be imaged onto the normally appropriate opaque spot on the cutoff grid and will instead pass through a transparent point and continue on to the final image plane. That image plane is located a distance from the camera lens such that it is the conjugate plane of the test region, and thus light areas of the final image correspond to areas in the test region in which light was deviated from its original course. This is in general more of a visualization or a qualitative tool rather than a quantitative measurement of refractiveindex variations.

Advantages of the focusing schlieren system over a conventional knife-edge schlieren system using collimated light include the ability to use an extended light source, the possibility of the test-region area of field being significantly larger than the area of the camera lens, and a small depth of focus. This last property means that, by moving the final image plane, we can select different parts of the test region on which to focus. Another advantage is that the conventional system requires that the lenses or mirrors used be of very high optical quality to eliminate aberration effects from appearing in the schlieren image, whereas the focusing system can be designed to relax this requirement significantly. The focusing schlieren system can also be modified in various ways. For example, a Fresnel lens is often placed just before the source grid to concentrate the light source into the imaging-lens aperture, thus producing a high-brightness final image. Similarly, the direction of sensitivity can be controlled by the type of grid used in source-grid and cutoff-grid planes. A grating pattern (horizontal or vertical) can be used for sensitivity in just one direction, or a checkerboard pattern can allow sensitivity in both orthogonal directions simultaneously. The period of the grating or the checkerboard pattern determines the absolute sensitivity as given by a minimum angle of deviations that can be detected. A good review of focusing schlieren systems and their characteristics is found in a recent paper by Weinstein. ${ }^{9}$

The purpose of this paper is not to review in detail the concept of focusing schlieren systems but to demonstrate how bacteriorhodopsin films may find application in such a system. In previous systems one usually produced the cutoff grid by placing a sheet of high-contrast photographic film in the cutoff location and exposing it to the image of the source grid. The developed film was then an exact negative of the source grid and was placed back into position in the schlieren system. Making sure the cutoff grid was positioned correctly was critical to system performance, and realignment could be difficult, as it was necessary for it to be repositioned accurately in the $x$, $y$, and $z$ coordinates, as well as for rotation. In this sense it is clear that a real-time film that does not require removal and external developing would be highly advantageous. Bacteriorhodopsin films have this property of instantaneous developing upon exposure to light, and it can be made to behave as a negative with the proper choice of exposing and reading wavelengths. In addition to facilitating system setup and alignment, a real-time film also allows adaptation in that the source- and cutoff-grid patterns may be changed at will to vary orientation or absolute sensitivity. It may even permit use of an unstructured scene as a grid that varies over time, as the cutoff grid could be easily updated to keep up with a changing source grid.

\section{B. Bacteriorhodopsin Films}

BR is a protein molecule found in the photosynthetic system of a salt-marsh bacterium called Halobacterium salinarium. The BR molecule is located in a cell membrane commonly called the purple membrane. To the bacterium, BR is important in an oxygendeficient environment, as the BR molecules function as light-driven proton pumps that transport protons across the cell membrane. This creates a proton gradient, which in turn generates an electrochemical potential used by the organism to synthesize adenosine triphosphate. Effectively, BR is used by the bacterium to convert sunlight directly into chemical energy. The absorption of light also initiates a photocycle in the BR molecule in addition to the transportation of protons. The characteristics and effects of this photocycle make it a potentially useful material as an optically addressed spatial light modulator. This is especially true because the photocycle continues to function after the BR material is extracted in the form of two-dimensional crystalline sheets of purple membrane and suspended in various solutions for the preparation of films. 
A schematic diagram of the BR photocycle is shown in Fig. 2. In the dark the molecule is initially in the $b R$ state, which has an absorption spectrum peaked at $\sim 570 \mathrm{~nm}$. Upon absorption of a photon in this band, the molecule quickly passes through several intermediate states by means of thermal relaxation until it reaches the $M$ state, in which its absorption spectrum is shifted $\sim 160 \mathrm{~nm}$ toward the blue from the initial $b R$ state. In this state the peak-absorption wavelength lies at $\sim 410 \mathrm{~nm}$. Thus images may be written in a film of BR based on the conversion of molecules from the $b R$ state to the $M$ state. In its native form, at nominal $p \mathrm{H}$, relative humidity, and ambient temperature, the lifetime of the $M$ state is $\sim 10 \mathrm{~ms}$. However, the image lifetime can be increased by several orders of magnitude by use of chemical $^{2}$ and mutagenic ${ }^{10}$ methods. The $M$ state can also be stabilized by temperature control at approximately $-40{ }^{\circ} \mathrm{C} .^{2}$ Additionally, one can actively erase images by exposing the material in the $M$ state to blue light, which directly returns the molecule to the $b R$ state. Thus BR films can effectively be used as real-time, erasable optical photographic or holographic films that require no development process, although in general the images recorded are not permanent. Other characteristics of importance for the focusing schlieren application include a potentially fast image-writing rate limited to $\sim 50 \mu$ s by the intrinsic photocyle time and also very high spatial resolution of several thousands of line pairs per millimeters because of the small molecular size of BR. The effective contrast for transmission between material in the $b R$ and the $M$ states depends principally on the BR concentration in the film solution, the $M$-state lifetime, and the wavelength of light, and we investigate this further in Section 3.

The film used in this experiment was prepared from a genetic variant of the wild-type form of BR by Bend Research, Inc. The molecule is called the $\mathrm{D} 96 \mathrm{~N} / \mathrm{T} 46 \mathrm{~V}$ variant and is genetically created by replacement of an aspartic acid with asparagine and a theonine with a valine. This variant was chosen for its extended image lifetime, as the genetic substitutions apparently affect the tertiary structure of the $\mathrm{BR}$ molecule sufficiently to change the $p \mathrm{H}$ depen-

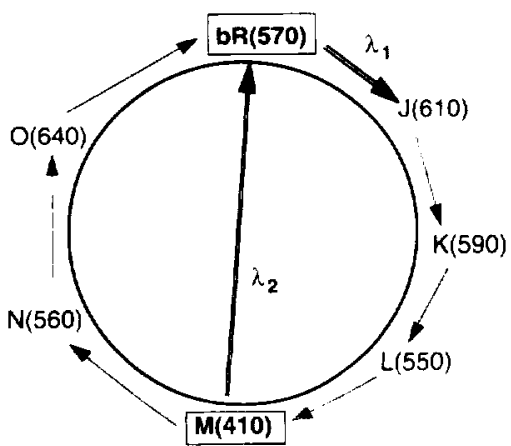

Fig. 2. Schematic of the BR photocycle. Numbers in parentheses indicate the peak wavelengths of absorption spectra at the $b R$ and the intermediate states. dence of the $M$-state lifetime. After extraction from the bacteria, the purple-membrane material was suspended in a $10 \%$ gelatin polymer matrix and was deposited on an optically flat substrate. The film was dried in a controlled humidity environment to lower the water content to a value of $\sim 20 \%$. A sodium hydroxide additive was employed in the solution to raise the solution $p \mathrm{H}$ to $\sim 9.0$, which served to lengthen the $M$-state lifetime (image lifetime) to several minutes. After drying, the film was sealed between the substrate and another optically flat glass plate to ensure a uniform thickness across the aperture of 1-in. (2.54-cm) diameter. The film thickness is $\sim 100 \mu \mathrm{m}$, and the optical density of the film in the $b R$ state at the peak wavelength of the absorption spectrum is 4.5 .

\section{Schlieren Techniques with Bacteriorhodopsin}

The proposed focusing schlieren system using bacteriorhodopsin is thus very similar to the general type shown in Fig. 1 with the exception that a BR film is placed in the system at the location of the cutoff grid. There are two basic methods whereby one can create a cutoff grid and then perform the schlieren test. In both cases we need light at two wavelengths or bands, either from two different sources or obtained by use of spectral filters with a broadband white-light source. The two bands required are in the yellow/green, preferably centered around the $b R$-state absorption maximum of $570 \mathrm{~nm}$, and in the blue, preferably centered around the $M$-state absorption maximum of $410 \mathrm{~nm}$. However, given the relatively broad absorption spectra exhibited by the BR states, one can use wavelengths not at the peaks and still obtain reasonable contrast and performance.

The first method is very straightforward. We begin with the BR film uniformly dark, or in the ground $b R$ state. We then illuminate the source grid with a yellow or a green source, or, if we are using a white-light source, we place a yellow spectral band filter in front of the BR film during imaging. The source grid is thus imaged onto the BR film in yellow light, causing the exposed areas to bleach, or convert predominantly to the $M$ state. This produces an image of the source grid in the BR film. To read out, we use a weak blue-light source to illuminate the source grid or exchange a blue spectral filter for the yellow if we are using a white-light source. In the absence of any phase or refractive-index variations during readout, blue light transmitted by the transparent areas of the source grid is imaged onto regions of the BR film that are predominantly in the $M$ state, and the opaque areas of the source grid are imaged onto regions of the film that are still in the $b R$ state. Because the $M$ state is highly absorptive to blue light whereas the $b R$ state is relatively transparent, the image written in the BR film by yellow light acts essentially as the negative of the source-grid image read out in blue light. Therefore in the absence of refraction in the test region, the final image should be uniformly dark or dim because most of the blue light 
will be absorbed. When refractive-index variations are present in the test region, then some of the light will be deviated onto parts of the BR film in the original $b R$ state and transmitted because of the lower absorption level of this state. The image will then contain light areas that correspond to these portions of the test region, just as in commonly used focusing schlieren systems with fixed photographic negative cutoff grids.

The second method is very similar to the first except that we reverse the roles of the yellow and the blue sources during cutoff-grid recording and readout. Assuming the BR film is uniformly in the ground $b R$ state initially, we first flood the entire film with strong uniform yellow light. This step is to bleach the entire film, converting the whole area predominantly to the $M$ state. This of course is absorptive to blue light and transmissive to yellow light. To record the cutoff grid, we illuminate the source grid with a strong blue-light source. The blue image at the BR film plane causes photoconversion of the illuminated areas back to the ground $b R$ state. We then perform readout with a weak yellow source illuminating the source grid. In the absence of phase or refractive-index variations during readout, yellow light transmitted by the clear areas of the source grid is absorbed by those regions of the BR film that are in the $b R$ state, and the opaque areas of the source grid are imaged onto regions of the film that are still predominantly in the $M$ state. Thus the image recorded in the film with blue light acts as a negative to the readout with yellow light, and the system will behave as a focusing schlieren optical system.

\section{Experiments and Results}

To perform demonstration experiments of a focusing schlieren system with a BR film as an adaptive cutoff grid, I used an argon laser $(\lambda=514.5 \mathrm{~nm})$ and a He-Cd laser ( $\lambda=442 \mathrm{~nm}$ ) as the two light sources that fall within the absorption spectra of the $b R$ state and the $M$ state, respectively. These choices were made for reasons of availability and laser power. The wavelengths are not at the peaks of the absorption spectra, but as noted earlier, this is not an impediment. Given these two light sources, the first step in designing the system was to decide on one of the two methods just discussed, i.e., which source to use to record the cutoff grid and which to use for readout during the schlieren testing. The important information for making this decision is obtained by observation of the transmission-level contrast experienced by each of the two wavelengths between material in the $b R$ and $M$ states. Therefore the first experiment was to perform these measurements.

\section{A. Contrast Measurements}

The light-transmission characteristics of the D96N/T46V film at both wavelengths were measured experimentally with a strong bleach, or write, beam and weak read beams whose intensities were small enough that they would not significantly contribute to the photoconversion and thus not affect the transmission measurements. A schematic of the experimental setup is shown in Fig. 3. The write, or bleach, beam is an argon laser beam at $514.5 \mathrm{~nm}$ with a power density of $\sim 30 \mathrm{~mW} / \mathrm{cm}^{2}$ at the BR-film plane. Figures $4(\mathrm{a})$ and $4(\mathrm{~b})$ show the transmission levels of the $514.5-\mathrm{nm}$ and $442-\mathrm{nm}$ wavelengths as a function of time, respectively. In the experiments the BR film initially starts out completely in the ground $b R$ state. At $t=10 \mathrm{~s}$ the bleaching beam is turned on, and at $t=30 \mathrm{~s}$ it is turned off again. Thus the transmission curves demonstrate behavior of both the photoconversion and the thermal-decay processes for this film. For the focusing schlieren application we are mainly interested in the contrast in transmission between the ground $b R$ state and the $M$ state, or even more importantly, the mixed population that is found sometime after the writing beam is turned off.

Consider first the curve in Fig. 4(a) for the 514.5-nm read beam. Recall that we are comparing the transmission of the bleached or semibleached film to the original $b R$ state, and we see that the maximum contrast available is more than $26: 1$ and would be even greater if we either left the bleaching beam on longer or just used a stronger bleach beam. However, a more realistic measurement is the contrast obtained some time after the bleach beam is turned off. For example, $15 \mathrm{~s}$ after writing $(t=45 \mathrm{~s})$, the contrast is $\sim 10: 1$, and after $30 \mathrm{~s}(t=60 \mathrm{~s})$, it is still greater than 8.5:1. On the other hand, the data in Fig. 4(b) show that the maximum contrast available with a blue read beam at $442 \mathrm{~nm}$ is $\sim 4.5: 1$, and it drops to $2.6: 1$ and $2.3: 1$ after $15 \mathrm{~s}$ and $30 \mathrm{~s}$ have elapsed since the write beam was turned off, respectively. Measurements of the absolute transmission levels indicated that the relatively low contrast at 442 $\mathrm{nm}$ is generally caused by a higher background absorption at this wavelength by the $b R$ state. These results make it clear that a read beam at the green wavelength will experience far greater contrast in a written image in the film than the blue wavelength and that the contrast to the green is significant both immediately after writing and for quite awhile afterward.

\section{B. Schlieren Experiments}

Given the results of the transmission measurements of the BR film at the argon and the $\mathrm{He}-\mathrm{Cd}$ laser

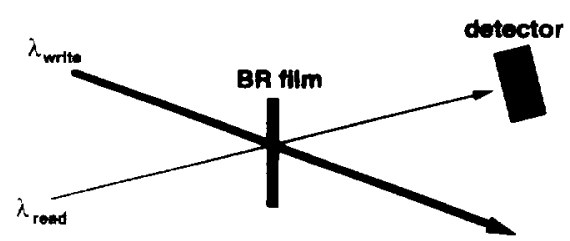

Fig. 3. Schematic of the optical setup for making measurements of transmission characteristics of BR film. The wavelength of the write beam was $514.5 \mathrm{~nm}$, and the wavelength of the readout beam was alternately $514.5 \mathrm{~nm}$ and $442 \mathrm{~nm}$. 


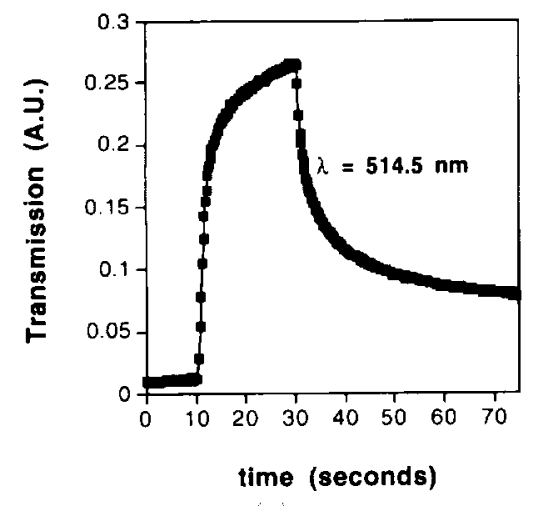

(a)

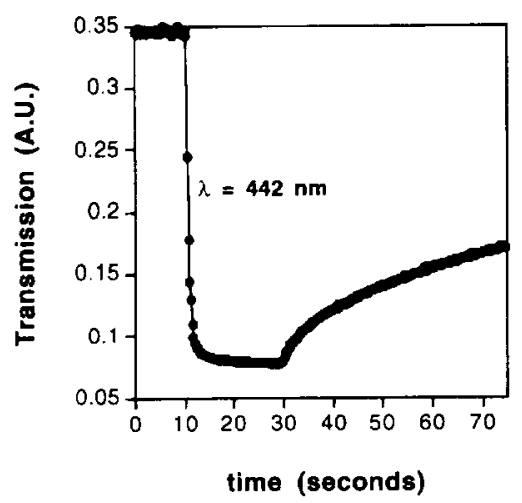

(b)

Fig. 4. Results of transmission experiments at (a) $514.5 \mathrm{~nm}$ and (b) $442 \mathrm{~nm}$. In both cases the bleaching or writing beam was turned on at $t=10 \mathrm{~s}$ and switched off at $t=30 \mathrm{~s}$.

wavelengths, it is apparent that the 514.5 -nm wavelength experiences greater contrast between the two $\mathrm{BR}$ states, and thus the image of the source grid recorded by the film will be much closer to a true negative at this wavelength. I therefore implemented the second technique described for the BR schlieren system. That is, I used the blue wavelength for writing the cutoff grid and the green wavelength for readout through the test region. The schlieren optical system used in the experiments is shown in Fig. 5. An argon-ion laser beam at a wavelength of $514.5 \mathrm{~nm}$ is collimated and then split into two arms with a nonpolarizing beam splitter, preserving the vertical linear polarization in each beam. One beam is the bleaching beam, which is used to illuminate the entire BR film initially to photoconvert the BR molecules uniformly from the ground $b R$ state to the $M$ state. The collimated blue $\mathrm{He}-\mathrm{Cd}$ beam then illuminates the source grid, which is imaged onto the BR film. The blue image photoconverts the illuminated areas of the film back to the ground $b R$ state, leaving the rest of the film in the $M$ state. This step effectively creates the cutoff grid. Finally, the last step is the readout phase with the second argon beam and with a test object or flow in the test region. The readout beam is weakened with neutral-density filters (not shown in Fig. 5) so as not to alter significantly the BR-state distribution in the film. This beam passes through a ground-glass dif-

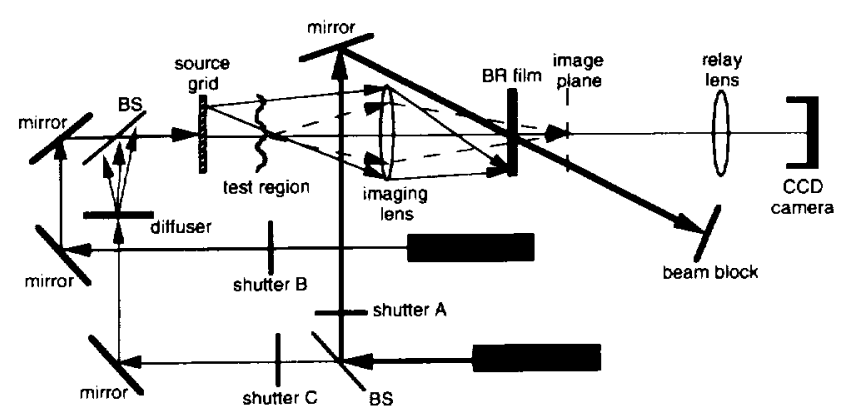

Fig. 5. Optical setup for focusing schlieren experiments with bacteriorhodopsin film: BS's, beam splitters; $\mathrm{Ar}^{+}$laser, argon-ion laser.

fuser to create effectively an extended source, reflects from a beam splitter, and uniformly illuminates the source-grid transparency. This light then passes through the test region, and some of the refracted light passes through BR film, where the material is in the transmissive $M$ state, and on to the image plane of the test-region slice. In our setup we use a second lens to relay the image plane into a CCD camera. All three of the beams are individually controlled with electronic shutters.

The source grid used in the schlieren experiments was a $35-\mathrm{mm}$ film-transparency grating pattern with equally spaced horizontal transparent and opaque lines. The grating frequency was 2.9 lines $/ \mathrm{mm}$. The source-grid area and the test-region field of view were both $\sim 1$ in. $^{2}\left(2.54 \mathrm{~cm}^{2}\right)$. This schlieren system was designed not to be a practical working system for most test objects but to provide an adequate system to study the feasibility and limitations of using a BR film as an adaptive cutoff grid. The power level of the argon laser before spatial filtering and collimation was $200 \mathrm{~mW}$, and the power level of the $\mathrm{He}-\mathrm{Cd}$ laser was $\sim 70 \mathrm{~mW}$. A neutral-density filter with an optical density of 3 was placed in the path of the readout argon beam. The magnification of the source grid as imaged by the imaging system onto the $\mathrm{BR}$ film was $\sim 0.42$. Under these conditions the initial bleaching step with the strong argon beam required $\sim 1 \mathrm{~s}$, the writing of the cutoff grid with the $\mathrm{He}-\mathrm{Cd}$ beam required $\sim 10 \mathrm{~s}$, and readout with the weak argon beam was possible for several minutes owing to the long $M$-state lifetime of the $\mathrm{D} 96 \mathrm{~N} / \mathrm{T} 46 \mathrm{~V}$ variant film. After this period of readout time, when the schlieren image began to darken, I could refresh the system by repeating the bleaching and the cutoff-grid writing steps and continue to view the same or a new test object.

Figure 6 shows the output image of the system in the absence of a test object. In this case the source grid illuminated in green light during readout is imaged exactly onto its negative encoded in the BR film, and thus most of the light is absorbed by the film and the image is fairly uniformly dark, as expected. The first test object studied was a simple positive lens of focal length $1000 \mathrm{~mm}$. The purpose of this test object was simply to demonstrate that the observed 


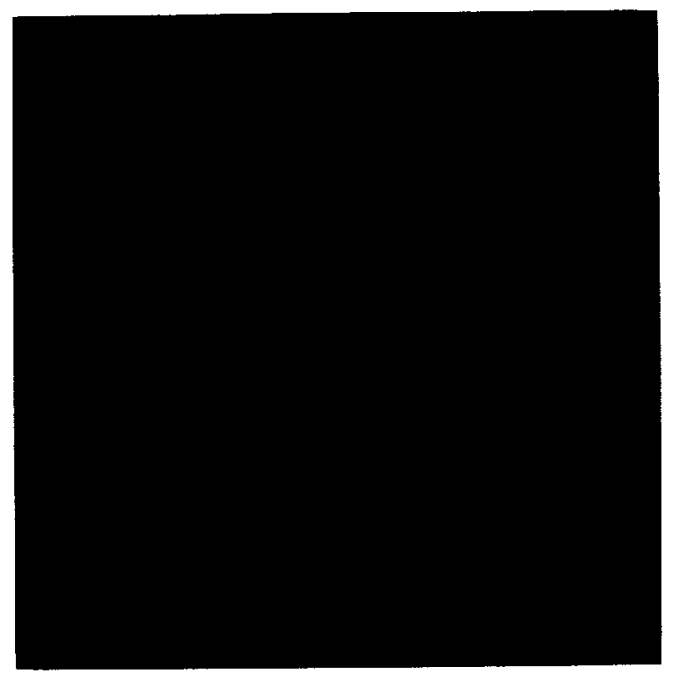

Fig. 6. Schlieren output image obtained in the absence of phase aberration in the test region.

effect was indeed the schlieren effect. Recall that the transmission function of a lens is essentially given by

$$
t(x, y)=\exp \left|-j \frac{\pi}{\lambda f}\left(x^{2}+y^{2}\right)\right|,
$$

where $f$ is the focal length of the lens. The grid used in the schlieren system is horizontal, and thus only refraction in the $y$ direction will allow light to pass through the transmissive areas in the cutoff grid. Because $t(x, y)$ in Eq. (1) is a separable function, we should expect that the schlieren pattern is constant in the $x$ direction and varies only in the $y$ direction. The observed schlieren pattern is shown in Fig. 7 . The central dark band corresponds to the central part of the lens, for which $y=0$, and thus no $y$ refraction occurs and the light is blocked at the BR-film plane. The first light bands on either side correspond to

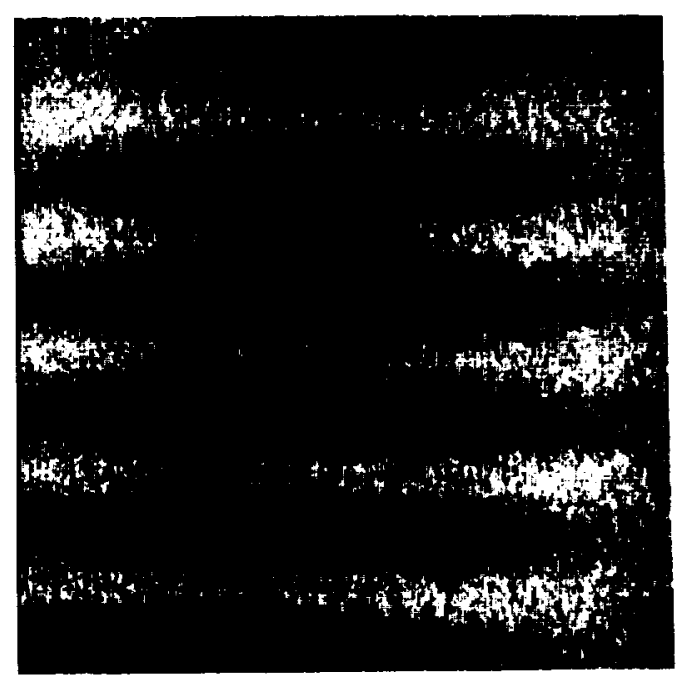

Fig. 7. Schlieren output image of a $1000-\mathrm{mm}$ positive lens in the test region. those same regions of the lens, which provide just enough refraction to deviate the light passing through them to displace its position at the BR-film plane by half of a grating period, i.e. from an opaque line to a transmissive line. Similarly, the next dark bands correspond to a displacement at the cutoff grid of one full period, and so on. The image agrees with what we would expect from a focusing schlieren system. The speckled nature of the image, of course, results from our use of a coherent laser during readout that has passed through the rough diffuser.

I then tested two more realistic objects using the same BR schlieren system. The first was a small low-velocity air jet. The image obtained from this test is shown in Fig. 8, in which the stream is clearly visible emanating from the nozzle against the dark background. Although it is not shown here, I also tested the air jet when it was pointed in the vertical direction. In this case the jet was virtually invisible, indicating that the flow is quite smooth and acts to refract light only perpendicular to the flow (in the $y$ direction in Fig. 8) and not in the direction of the flow (in the $x$ direction in Fig. 8). The second test object was a small clear plastic plate under stress shown in Fig. 9. It shows up quite clearly, and the stress distribution in the $y$ direction can be discerned very easily by the light pattern, especially evident in the lower right-hand corner of the plate.

\section{Discussion}

I have demonstrated the application of bacteriorhodopsin films to an adaptive focusing schlieren optical system for the visualization of refractive-index variations. In the experiments I used a film made from the $\mathrm{D} 96 \mathrm{~N} / \mathrm{T} 46 \mathrm{~V}$ BR variant because of the significantly extended image lifetime of this variant. The technique employed here was first to bleach the entire film with green/yellow light, then write the cutoff grid in the film with a strong blue source, and then read out with a green source so that the cutoff grid was the negative of the green image of the source grid.

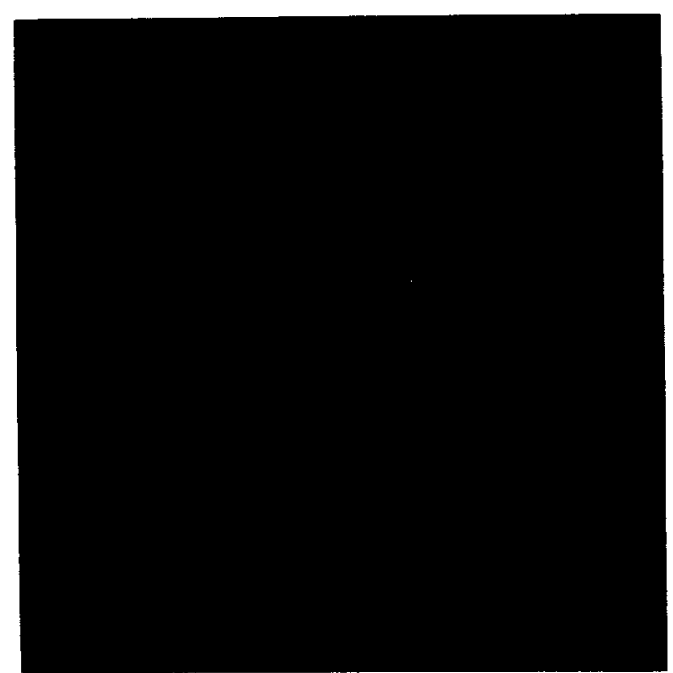

Fig. 8. Schlieren out put image of a small air jet in the test region. 


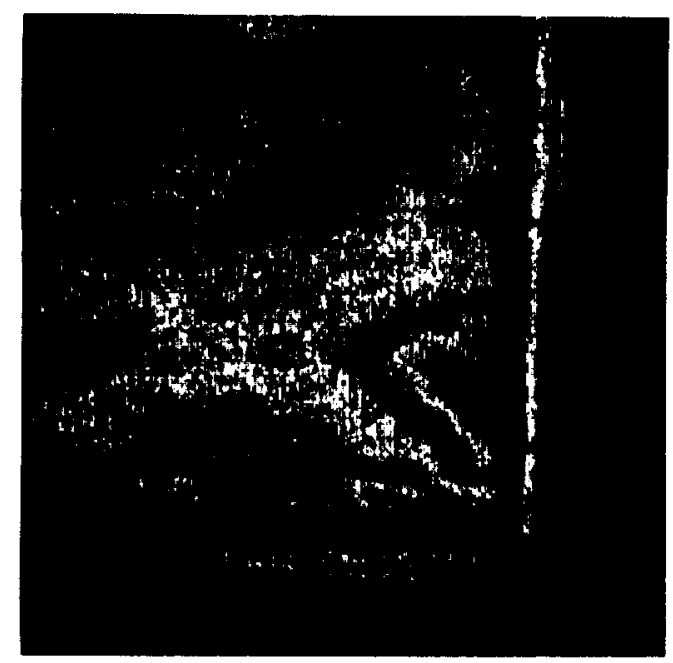

Fig. 9. Schlieren output image of a stressed clear plastic plate in the test region

This approach was chosen because of the significantly higher contrast experienced by the green wavelength in comparison to a blue readout beam. The $\mathrm{BR}$ schlieren system offers several potential advantages with respect to previous focusing schlieren systems. The first is ease of alignment, as the cutoff grid is developed in situ in real time in the BR film. In comparison, a conventional photographic film used to create the cutoff grid must be removed for development and then carefully replaced back in the optical system in precisely the same position as when exposed. The second and more important advantage is the potential for adaptivity. Because the cutoff grid written in the BR film can be erased and rerecorded at will, one can easily change the source grid and correspondingly write a new cutoff grid to accommodate system changes such as absolute sensitivity (changing the grating period) or sensitivity orientation (changing the orientation of the source grid) or both. These adaptations could be performed as quickly as one could change the source grid and would not require any system downtime to accomplish. One can even envision encoding the source grid on an electrially addressed spatial light modulator, which would facilitate very fast change rates.

On the other hand, these are also some disadvantages and limitations to the proposed BR system. The main one is that it requires two sources corresponding to the absorption spectra of the $b R$ and $M$ states of the bacteriorhodopsin photocycle: one in the yellow/green and one in the blue, respectively. In my proof-of-concept experiments I used an argonion laser at $514.5 \mathrm{~nm}$ and a He-Cd laser at $442 \mathrm{~nm}$. Although this type of setup may be acceptable for some situations, one would probably prefer to use a broadband white-light source and interchangeable yellow and blue bandpass filters so as to require only one source and to avoid speckle effects in the schlieren images. I attempted such an implementation experimentally but was unsuccessful for a couple of reasons. For the second approach (bleach uniformly, write the cutoff grid with blue light, readout with green/yellow light) I found that the available white-light sources simply did not have sufficient power in the blue portion of the spectrum to photoconvert the BR molecules from the $M$ state to the $b R$ state in numbers great enough to write a well-defined cutoff grid in a short period of time. For the first approach (start with unbleached film, write the cutoff grid with yellow light, readout with blue light), writing the cutoff grid proved not to be a problem, as the yellow/green power was adequate. However, the contrast level of the cutoff grid as seen by the blue readout light was too low to achieve satisfactory performance. This is caused mainly by a relatively high residual absorption in the blue of the ground $b R$ state. To avoid these problems when using a single broadband source, one may need to use a source with a significantly bluer spectrum to implement the second approach described. An alternative that might allow either approach would be the development of a material that exhibits a long-lived red shift rather than the blue shift found in the $M$ state. One possibility might be a BR mutant with a longer-lived $O$ state (absorption spectrum at $\sim 640 \mathrm{~nm}$ ) and a short M-state lifetime. This has in fact been observed in the variant $L 93 \mathrm{~T}$, in which the $O$-state lifetime was measured at $\sim 800 \mathrm{~ms}$ (Ref. 11) with an $M$-state lifetime of $\sim 30 \mathrm{~ms}$ (Ref. 12). Further study would of course be necessary to determine if this or another variant could be easily made into highquality films with the appropriate sensitivity and other necessary characteristics for successful application to this type of system.

This work was supported by the NASA Office of Advanced Concepts and Technology under RTOP 233-02-05-06. The BR film was made by Bend Research Inc., Bend, Oregon. The author thanks Dan Smithey for helpful discussions and Robert Youngquist for suggesting the idea.

\section{References}

1. C. Bräuchle, N. Hampp, and D. Oesterhelt, "Optical applications of bacteriorhodopsin and its mutated variants," Adv. Mater. 3, 420-428 (1991).

2. R. R. Birge, "Photophysics and molecular electronic applications of the rhodopsins," Ann. Rev. Phys. Chem. 41, 683-733 (1990).

3. R. Thoma, N. Hampp, C. Bräuchle, and D. Oesterhelt, "Bacteriorhodopsin films as spatial light modulators for nonlinearoptical filtering," Opt. Lett. 16, 651-653 (1991).

4. Q.W. Song, C. Zhang, R. Gross, and R. Birge, "Optical limiting by chemically enhanced bacteriorhodopsin films," Opt. Lett. 18, 775-777 (1993).

5. T. Renner and N. Hampp, "Bacteriorhodopsin-films for dynamic time average interferometry," Opt. Commun. 96, 142$149(1993)$.

6. N. Hampp, R. Thoma, D. Oesterhelt, and C. Bräuchle, 'Biological photochrome bacteriorhodopsin and its genetic variant ASp96 - Asn as media for optical pattern recognition," Appl. Opt. 31, 1834-1841!1992). 
